\title{
The Potential Role of a Health and Demographic Surveillance System in Rural Northern Nigeria to Reduce Maternal and Child Deaths
}

\author{
Olatunji Alabi1,2*, Henry V. Doctor ${ }^{3}$ \\ ${ }^{1}$ Nahuche Health and Demographic Surveillance System, Zamfara State, Nigeria \\ ${ }^{2}$ Demography and Social Statistics, Federal University, Birnin Kebbi, Nigeria \\ ${ }^{3}$ Regional Office for the Eastern Mediterranean, Department of Information, Evidence and Research, \\ World Health Organization, Cairo, Egypt \\ Email: vomistorii@yahoo.com
}

Received 21 November 2015; accepted 26 December 2015; published 29 December 2015

Copyright (C) 2015 by authors and Scientific Research Publishing Inc.

This work is licensed under the Creative Commons Attribution International License (CC BY). http://creativecommons.org/licenses/by/4.0/

(c) (i) Open Access

\section{Abstract}

There exist a huge gap between generation of evidence-based research findings and its use to inform policies in most developing countries. Further, it is evident that most developing countries are lagging behind in formulating appropriate policies aimed at improving people's lives due to lack of evidence-based research findings. We describe the potential of a Health and Demographic Surveillance System (HDSS) in informing appropriate health interventions towards reducing the high maternal and child deaths in rural communities of north western Nigeria through the Verbal Autopsy (VA) data collection. VA data collection involves the use of VA questionnaires-set of open ended and closed ended questions adapted from the World Health Organization (WHO) moduleadministered to the caregivers, parents or family members of a deceased person to elicit information on signs and symptoms and their durations, and other pertinent information about the deceased in the period before death. VA interviews were conducted by trained VA enumerators on all 2100 deaths reported during the update round 4 of routine data collection (July-December, 2012) and returned forms were checked for consistencies and completeness by a trained research officer. The forms were later coded by trained medical doctors for possible cause of death using the WHO International Classification of Diseases (ICD 10) codes. Fifty cases of neonatal deaths, 1650 cases of infant and child deaths, and 400 cases of adult deaths were reported during the update round 4 data collection. Neonatal sepsis was reported as the leading cause of neonatal deaths $(58 \%)$ while malaria and intestinal infectious diseases were reported as the leading cause of infant and child deaths and adult deaths respectively ( $45 \%$ and $17 \%$, respectively). The study provides documented evidence of high neonatal deaths due to neonatal sepsis in an area with low hy- 
giene and high home delivery rates. The findings from the VA data collection at Nahuche HDSS inform the intervention study on home distribution of chlorhexidine to pregnant women. The findings from this study call on government and other stakeholders to strengthen research capacity to generate timely data and findings returned to policy makers within the shortest period of time for decision making.

\title{
Keywords
}

\author{
Maternal and Child Deaths, Verbal Autopsy, Chlorhexidine, Demographic Surveillance, Health \\ Systems, Nigeria
}

\section{Introduction}

Issues related to maternal and child health remain one of the most common topics on all national and international foras aimed at addressing the poor maternal and child health indicators in most developing countries. Early into the Millennium, efforts at addressing developmental issues in general and poor maternal and child health in particular culminated into what was known as the Millennium Development Goals (MDGs) 5 and 4 respectively. Barely one year to the end of the MDGs, the battle against high regime of maternal and child health in most developing countries remains insurmountable despite some appreciable gains in the reduction of child and maternal deaths in most of these countries [1]. The continued high regime of maternal and child deaths will continue to feature in development discussions at different platforms for yet another 15 years through the proposed Sustainable Development Goal 3 targets 3.1 and 3.2 (SDG) by the United Nations as a follow up to the MDGs [2].

In 2013, nearly 17,000 under-five deaths were reported daily in the world despite a huge reduction in underfive deaths compared to the 1990 figures [3]. One striking revelation is that almost 9000 of the under-five deaths occur in only five countries daily and; India and Nigeria are responsible for almost 6000 under-five deaths per day in the world [3]. In Nigeria for instance, maternal mortality ratio was estimated at 576 deaths per 100,000 live births in 2013 while under-five mortality was estimated at 128 deaths per 1000 live births [4].

Within Nigeria, there is a variation in mortality rates between regions and place of residence. For instance, infant mortality rates are higher in the rural area (86 deaths per 1000 live births) than in the urban area (60 deaths per 1000 live births) and also highest in the North West geo-political zone (89 deaths per 1000 live births compared with 61 deaths per 1000 live births in the South west zone) [4]. Although, there had been a decrease of 31 percent in infant mortality rates in Nigeria over a 10-year period (2003-2013) from 100 to 69 deaths per 1000 live births, the current mortality rates continued to raise concerns among stakeholders in the health sector particularly as we drew closer to the 2015 deadline of the MDGs.

Addressing the challenges associated with high child and maternal mortality in Nigeria is compounded by the fact that most of these deaths occur at homes and thus, medical certification and obtaining accurate data on counts and causes to inform interventions in resource constraints communities like northern Nigeria remains elusive [5]. Continuous enumeration of births, deaths and migration- the core objective of a health and demographic surveillance system (HDSS) — and the capacity to determine the probable cause of deaths through Verbal Autopsy (VA) data collection is a possible response to address this challenge.

The aim of the paper is to highlight the importance of VA data collection in an HDSS setting and its potential in informing appropriate health interventions towards reducing the high maternal and child deaths in rural communities of north western Nigeria. The study is therefore timely and important at providing policy direction towards reducing maternal and child deaths in Zamfara State in particular and north-west Nigeria in general.

\section{Methodology}

\subsection{Study Area}

The Nahuche Health and Demographic Surveillance System (Nahuche HDSS) currently operates in six districts of Bungudu emirates in Zamfara State, north west Nigeria. The HDSS covering more than 100 villages in three 
political wards was established to provide a platform for research to generate evidence-based intervention and policies aimed at improving maternal and child health. In addition, the HDSS tracks population changes through detailed longitudinal socio-demographic and health data collection of demographic events of a dynamic cohort since 2009.

Nahuche HDSS was a collaboration between the Partnership for Reviving Routine Immunization in Northern Nigeria; Maternal Newborn and Child Health (PRRINN-MNCH) Programme-the Department for International Development (UKaid) and Norwegian Government Funded Programme and the Zamfara State Government. The study area, (Nahuche-keku, Nahuche-Ubandawaki, Gada, Bela, Rawayya and Karrakai districts) is a typical rural northern Nigeria setting where infrastructure remains deficient with inadequate power supply. The area is also characterized by poor road networks, which became virtually inaccessible during rainy season, and underdeveloped sanitation system [6].

The population of the surveillance area was 125,149 (62,389 males and 62,760 females) individuals during the baseline census conducted in 2010 [7]. After four update rounds (biannual data collection exercise), the population of the DSA increased to 137,823 (70,300 males and 71,827 females) from over 21,000 households in 2012. The under-five mortality rate of the DSA was 224.8 per 1000 live births and maternal mortality estimated for the surveillance area through the sisterhood method was 1049/100,000 live births (95\% CI, 1021 - 1136) in 2012 [7]. Anecdotal evidence showed that malnutrition prevalence is high among the under-fives in the study area which may explain the sustained high under-five mortality rate.

\subsection{Verbal Autopsy Data Collection Process at Nahuche HDSS}

The Nahuche HDSS commenced operation with an initial census of the Demographic Surveillance Area (DSA) between September and December, 2010. After the baseline census, biannual update rounds of data collection to update demographic and health events data by trained enumerators commenced. Trained enumerators visited the households in 180-day work cycle to update both demographic and health events through the Household Registration Book (HRB) and reporting any new events to the Nahuche Health Research centre for computerization and processing. Ethical clearance for the study was obtained from the Zamfara State Research Ethics Committee (ZREC). Nahuche HDSS is also a member of the International Network for the Demographic Evaluation of Populations and Their Health (INDEPTH Network). Details of data collection, management and quality assurance process at Nahuche HDSS have been described elsewhere [8].

The importance of Verbal Autopsy (VA) data collection to determine the probable cause of death at any INDEPTH site cannot be over emphasized. VA data collection is a major pre-requisite for obtaining INDEPTH Network membership. VA questionnaires, the module of collecting VA data, are set of open ended and closed ended questions adapted from the WHO module administered to the caregivers, parents or family members of a deceased person to elicit information on signs and symptoms and their durations, and other pertinent information about the decedent in the period before death.

VA data collection at Nahuche HDSS commenced during round 4 update data collection in 2012. This was preceded by a training of 6 man team at Kitampo Health Research Centre in Ghana. The team was trained on VA data collection and management which include the basics of conducting a good VA interview, vetting of completed questionnaires for completeness and consistencies, data entry, generation of coding list from the computer centre, coding of completed questionnaires by trained clinicians, consensus coding in case of unresolved cause of deaths during independent clinician coding, data entry of coded questionnaires, and assignment of cause of death for each coded forms.

Furthermore, the team was trained on the required logistics for a smooth VA data collection and management. The team later trained VA data collection and management team on arrival back to Nigeria. Three VA data collectors were trained and assigned to the six districts under the demographic surveillance area (DSA) (i.e. 1 field worker per two districts since data are being collected only from households with reported cases of death). Deaths reported by the routine HDSS field workers were categorized into neonatal ( 0 - 27 days), infant and child (28 days - 12 years) and adult (13 years and above) deaths. The VA field enumerators revisit such households to elicit information on signs and symptoms and their durations, and other pertinent information about the deceased in the period before death from caregivers/parent/family members. Selection of appropriate respondent is key to a successful VA interview.

To ensure data quality, a research officer was trained to conduct spot checks and vetting of randomly selected 
completed questionnaires for completeness and consistencies in responses. The research officer then logged in all completed forms to the computer section for data entry through the filling clerk. At the computer section level, consistencies checks were in-built into the database to check errors not detected by the research officer at the field level. In case of such error, the questionnaire is returned back to the field for possible correction through the filling clerk to the research officer who then return such forms to the field worker.

Two sets of three medical doctors were trained on the data collection and management process involved as well as on the use of International Classification of Diseases (ICD 10) codes for assigning probable cause of death to ensure timeliness in data processes. To ensure quality of data at this level, a completed VA questionnaire is coded independently by three coders. In case of disagreement between the three coders on possible cause of death, a consensus coding exercise is carried out. This involves the three coders coming together at a round table to offer explanations as to why he/she assigned the previous codes as possible cause of death. The three will have to agree on a particular cause of death as the most plausible cause of death before such forms can be regarded as completed. Coded VA forms for possible cause of death (based on ICD 10 codes) are later entered into the Household Registration System (HRS2) software and data analysis carried out using the HRS2 software.

\section{Results}

A total of 2,100 deaths were reported during the update round 4 data collection at Nahuche HDSS. The VA data reported in this study were for update round 4 (July-December 2012). The deaths were disaggregated by age thus giving 50 cases of neonatal deaths, 1,650 cases of infant and child deaths and 400 cases of adult deaths. It is pertinent to point out at this level that the low level of coverage of neonatal deaths as evident in the number of cases of neonatal deaths reported was due to cultural and low literacy level within the DSA and efforts at improving the data such as the establishment of community key informant system (CKI) in all the districts for daily capturing of vital events like births and deaths in a timely way. The CKIs are mostly imams who incidentally are responsible for performing both birth and death rites. Details of efforts at improving on the data collection have been documented elsewhere [8].

Table 1 shows the percent distribution of deaths by cause of deaths reported during the update round 4 data collection. Neonatal sepsis was reported as the leading cause of neonatal deaths (58\%) while malaria and intestinal infectious diseases were reported as the leading cause of infant and child deaths and adult deaths, respectively ( $45 \%$ and $17 \%$, respectively).

Table 1. Percent distribution of top causes of death by age, Nahuche HDSS, 2012.

\begin{tabular}{|c|c|c|c|}
\hline Diagnosis & $\begin{array}{l}\text { Neonatal Deaths } \\
(0-27 \text { days })(n=50)\end{array}$ & $\begin{array}{c}\text { Infant and Child Deaths } \\
\text { (28 days - } 12 \text { years) }(n=1650)\end{array}$ & $\begin{array}{c}\text { Adult Deaths } \\
(13 \text { years and above) }(n=400)\end{array}$ \\
\hline Neonatal Sepsis & 58 & - & - \\
\hline Birth Asphyxia \& Birth Trauma & 28 & - & - \\
\hline Prematurity \& Low Birth Weight & 8 & - & - \\
\hline Other Disorders & 4 & - & - \\
\hline Neonatal Jaundice & 2 & - & - \\
\hline Malaria & - & 45 & 14 \\
\hline Intestinal Infectious Diseases & - & 26 & 17 \\
\hline Measles & - & 10 & - \\
\hline Pneumonia & - & 7 & - \\
\hline Malnutrition & - & 6 & - \\
\hline Cerebrovascular Diseases & - & - & 11 \\
\hline Hypertensive Heart Diseases & - & - & 6 \\
\hline Transport Accident & - & - & 6 \\
\hline
\end{tabular}

Note: The numbers in the table are percentages whereas the sample size is reported in the first row of the table. 


\section{Chlorhexidine and Misoprostol Intervention Study}

Neonatal sepsis has been documented as one of the leading causes of death in the first week of life particularly in resource constrained environment like northern Nigeria [9]. Incidence of neonatal sepsis ranges from $13 \%$ to $50 \%$ of newborn deaths [9] [10]. The high child mortality due to sepsis in resource constrained rural environment like Nahuche in Zamfara State in Nigeria is accounted for by many cultural practices of cutting and treatment of cord stump. It is usually a common scene to see traditional birth attendants cutting umbilical cords with objects like sharp stone, threads, used razor blades and unsterilized knives. Application of hot charcoal, herbal medication, vaseline and many other unhygienic substances to the newly cut cord also could well account for a high significant of newborn sepsis [10].

There are various researches on the efficacy of chlorhexidine in reducing neonatal deaths see, [9]-[12]. Studies from Nepal, Bangladesh and Pakistan showed that the application of chlorhexidine to the cord immediately after birth and up to two weeks reduces neonatal mortality significantly. For instance, a field study in Nepal showed that neonatal mortality was reduced by $24 \%$ when chlorhexidine was applied to the cord by community health workers during home visit [13]. A similar study from Pakistan showed a $42 \%$ drop in risk of newborn infection and almost $40 \%$ reduction in neonatal mortality when chlorhexidine is recommended and provided by TBAs to families [13].

Following the analysis of update round 4 VA data and proven evidence of efficacy of chlorhexidine in reducing neonatal mortality, the PRRINN-MNCH Programme in partnership with the USAID-funded Project Targeted States High Impact Project (TSHIP) piloted the most effective community based mechanism of distributing chlorhexidine and misoprostol to reduce child and maternal mortality due to neonatal sepsis and post partum hemorrhage. The study was conducted within the health development partnership between the two projects in order to test the hypothesis that distributing chlorhexidine and misoprostol at eight months of pregnancy reduces child and maternal deaths more than distributing the commodities during labour.

A total of 684 women for two exposure wards (A and B) were followed up for a period of 6 months. In exposure ward A, the pregnant women were given chlorhexidine and misorostol during labour while in ward B, pregnant women were given the commodities at 8 months of pregnancy. The analysis and findings from the study will be available soon.

\section{Conclusions}

Evidence-based interventions in public health are becoming clearer as the solution to most public health challenges in developing countries especially if the dividends of health research to determine which health systems reforms and programmatic combinations are better at achieving the goals of improved health and well-being are to be achieved [6]. Health and demographic surveillance systems are set up to generate evidence-based findings in a timely way to inform and influence population and health policies.

Verbal Autopsy data collection and management as demonstrated by Nahuche HDSS in Zamfara State, north-west Nigeria, are useful in populations that do not have a complete vital registration system to inform health interventions that will help reduce the high regime of child and maternal mortality in Nigeria in general and north-west Nigeria in particular to its barest minimum especially as we move into the post-Millennium Declaration. The findings from the VA data collection calls on government and other stakeholders to strengthen research capacity to generate data in a timely fashion and finding returned to policy makers within the shortest period of time for decision making.

\section{Limitation}

The study demonstrated how research findings can inform evidenced-based interventions that can inform policies at addressing the high child and maternal mortality in the study area. One important limitation is the unavailability of the result from the ongoing community-based distribution of chlorhexidine and misoprostol study. The data collection on the impact of the two commodities on child and maternal mortality reduction is ongoing. Also, the risk of misclassification of cause of death through the use of trained medical coders cannot be totally ignored.

\section{Acknowledgements}

We acknowledge the support received from the PRRINN-MNCH Programme (funded by DFID (UKaid) and the 
Government of Norway), both national and Zamfara State offices during the study. Also acknowledged are the leadership of Zamfara State Government, the chairman of Bungudu LGA and staff from Zamfara State Ministry of Health. The Nahuche HDSS supervisors, interviewers as well as the data entry staff and all the staff in various capacities who worked on the HDSS activities and this study are duly acknowledged. We also acknowledge the support of the six districts heads and community leaders and the respondents in particular who volunteered their time to respond to all the questions. The views expressed in this paper are those of the authors and do not represent the views of their organizations or institutions.

\section{References}

[1] Adedini, S.A. (2014) Neighbourhood Characteristics and Under-Five Mortality in Nigeria. African Population Studies, 27, 1-15. http://aps.journals.ac.za http://dx.doi.org/10.11564/27-2-474

[2] UNITED NATION Department of Economic and Social Affairs (2014) Open Working Group Proposal for Sustainable Development Goals. https://sustainabledevelopment.un.org/sdgsproposal

[3] World Health Organisation (2013) Global Health Observatory. www.who.int/gho/child health/mortality/mortality under five text/en/

[4] NPC and ICF International (2014) Nigeria 2013 Demographic and Health Survey. NPC and ICF International, Abuja and Rockville.

[5] Setel, P.W., Osman, S., Chalapati, R., et al. (2005) Sample Registration of Vital Events with Verbal Autopsy: A Renewed Commitment to Measuring and Monitoring Vital Statistics. Bulletin of the World Health Organization, 83, 611617.

[6] Alabi, O., Doctor, H.V., Afenyadu, G.Y. and Findley, S.E. (2014) The Lessons Learned from Setting up the Nahuche Health and Demographic Surveillance System in the Resource Constrained Context of Northern Nigeria. Global Health Action, 7, Article ID: 23368. http://dx.doi.org/10.3402/gha.v7.23368

[7] Doctor, H.V., Alabi, O., Findley, S.E., Afenyadu, G.Y., Abdulwahab, A. and Jumare, A. (2012) Maternal Mortality in Nigeria: Findings of a Health and Demographic Surveillance System in Zamfara State, Nigeria. Tropical Doctor, 42, 140-143. http://td.rsmjournals.com/content/early/recent http://dx.doi.org/10.1258/td.2012.120062

[8] Alabi, O., Doctor, H.V., Jumare, A., Sahabi, N., Abdulwahab, A., Findley, S.E. and Abubakar, S.D. (2014) Profile: The Nahuche Health and Demographic Surveillance System-Northern Nigeria [Nahuche HDSS]. International Journal of Epidemiology, 43, 1770-1780. http://dx.doi.org/10.1093/ije/dyu197

[9] Liu, L., Johnson, H.L., Cousens, S., et al. (2012) Global, Regional, and National Causes of Child Mortality: An Updated Systematic Analysis for 2010 with Time Trends Since 2000. The Lancet, 379, 2151-2161. http://dx.doi.org/10.1016/S0140-6736(12)60560-1

[10] Hodgins, S., Pradhan, Y.V., Khana, L., et al. (2013) Chlorhexidine for Umbilical Cord Care: Game-Changer for Newborn Survival? Global Health: Science and Practice, 1, 5-10. http://dx.doi.org/10.9745/GHSP-D-12-00014

[11] Imdad, A., Mullay, C.L., Baqui, A.H., et al. (2013) The Effect of Umbilical Cord Cleansing with Chlorhexidine on Omphalitis and Neonatal Mortality in Community Settings in Developing Countries: A Meta-Analysis. BMC Public Health, 13, S15. http://dx.doi.org/10.1186/1471-2458-13-S3-S15

[12] Saleem, S. (2013) Application of 4\% Chlorhexidine Solution for Cord Cleansing after Birth Reduces Neonatal Mortality and Omphalitis. Evidence-Based Medicine, 18, e15. http://dx.doi.org/10.1136/eb-2012-100752

[13] Wall, S. (2012) Fighting Newborn Infections: New Evidence Leads the Way. www.healthynewbornnetwork.org/blog/fighting-newborn-infections-new-evidence-leads-way 